\title{
Progress on vacuum-to-air mass calibration system using magnetic suspension to disseminate the Planck-constant realized kilogram
}

\author{
Eric C. Benck, Corey Stambaugh, Edward C. Mulhern, Patrick J. Abbott, Zeina J. Kubarych \\ Mass and Force Group, National Institute of Standards and Technology, 100 Bureau Dr., MS 8221, Gaithersburg, MD 20899, United States
}

\section{ABSTRACT}

The kilogram is the unit of mass in the International System of units (SI) and has been defined as the mass of the International Prototype Kilogram (IPK) since 1889. In the future, a new definition of the kilogram will be realized by fixing the value of the Planck constant. The new definition of the unit of mass will occur in a vacuum environment by necessity, so the National Institute of Standards and Technology (NIST) is developing a mass calibration system in which a kilogram artefact in air can be directly compared with a kilogram realized in a vacuum environment. This apparatus uses magnetic suspension to couple the kilogram in air to a high accuracy mass balance in vacuum.

\section{Section: RESEARCH PAPER \\ Keywords: magnetic suspension; mass metrology; Planck constant; revised SI; SI units; watt balance}

Citation: Eric C. Benck, Corey Stambaugh, Edward C. Mulhern, Patrick J. Abbott, Zeina J. Kubarych, Progress on vacuum-to-air mass calibration system using magnetic suspension to disseminate the Planck-constant realized kilogram, Acta IMEKO, vol. 6, no. 2, article 12, July 2017, identifier: IMEKO-ACTA-06 (2017)$02-12$

Section Editor: Min-Seok Kim, Research Institute of Standards and Science, Korea

Received June 30, 2016; In final form October 14, 2017; Published July 2017

Copyright: (C) 2017 IMEKO. This is an open-access article distributed under the terms of the Creative Commons Attribution 3.0 License, which permits unrestricted use, distribution, and reproduction in any medium, provided the original author and source are credited

Corresponding author: Eric C. Benck, e-mail: eric.benck@nist.gov

\section{INTRODUCTION}

The kilogram is the only remaining base unit in the International System of Units (SI) that is still defined by an artefact, the International Prototype Kilogram (IPK). The IPK is made of a platinum-iridium alloy and is maintained at the International Bureau of Weights and Measures (BIPM) in Sevres, France. Therefore the unit of mass can only be realized at the BIPM, and must be disseminated to the rest of the world through a chain of comparison calibrations by the world's National Metrology Institutes (NMIs). The NMIs maintain traceability to IPK through periodic comparisons of their $1 \mathrm{~kg}$ standard(s), which in most cases are also made of the same platinum-iridium alloy, with the working standards of the BIPM. The NMIs then realize a mass scale from approximately $1 \mathrm{mg}$ up to several thousand kilograms through multiple and sub-multiple calibrations involving their $1 \mathrm{~kg}$ standard(s) and working standard artefacts.

Redefinition of the kilogram based on Planck's constant will take place in 2018 [1], [2]. This will complete the redefinition of base units in the SI from artefacts to fundamental constants. When this happens both the watt balance and the International Avogadro Coordination (IAC) project will realize the kilogram in a vacuum environment [3], [4]. But most industry and research laboratories will continue to perform mass metrology in air, so at some point the unit of mass realized in vacuum must be transferred to masses in air. Consequently, methods of transferring the vacuum realization to atmospheric pressure will have to be developed [5].

Currently there are two approaches to establish traceability from the unit of mass realized in vacuum to the mass unit in air: sorption artefacts and a mass comparator utilizing magnetic coupling between air and vacuum [6].

The sorption artefact technique is based on accurately modelling the adsorption process of water and other contaminants from air. The mass of artefacts with similar mass but different surface areas are compared both in air and in vacuum. These measurements are then used to determine the adsorption coefficient of water and other contaminants from air 
per unit area. This is then used to calculate a correction factor which is simply added to a mass brought from vacuum into air. Measurement uncertainties on the order of several micrograms can be obtained with this technique [7].

The simplicity of simply adding a fixed mass correction value to a mass as it is removed from vacuum is a great advantage. But this is an indirect correction technique which depends on the accuracy of the adsorption model. The adsorption process is very sensitive to the surface state of the masses. It has been shown that adsorption coefficients depend on the artefact material, cleanliness, and surface roughness. Other factors such as oxide layer thickness and composition may also play an important role. Unsurprisingly, there is significant variations in values of reported sorption values [8], [9]. Great care is required to insure the surface state of masses remain identical to those involved in the adsorption modelling measurements.

The magnetic suspension technique, on the other hand, involves a direct comparison of a mass in vacuum with a mass in air. No assumptions based on the adsorption modelling need to be made. Figure 1 shows a schematic of the magnetic suspension mass comparator apparatus. In the upper chamber is a commercial mass comparator kept in vacuum on which masses in the upper chamber can be compared. A second weighing pan and carriage are magnetically suspended in the lower, air-filled chamber. This second weighing pan is connected to the mass comparator load cell by magnetic fields which penetrate through the vacuum-to-air boundary flange. This enables direct comparisons to be made between masses in vacuum in the upper chamber with masses in air in the lower chamber.

One challenge of the magnetic suspension technique is that in addition to the $1 \mathrm{~kg}$ mass, the mass comparator load cell must also support the upper and lower magnet assemblies, the air weighing pan and the associated support structures. Consequently, the mass comparator must be designed to handle a load significantly larger than $1 \mathrm{~kg}$. For the system described in this paper, the mass comparator has a maximum load of $10 \mathrm{~kg}$ and a resolution of $10 \mu \mathrm{g}$. It is the goal of this project to achieve an uncertainty of $20 \mu \mathrm{g}$ for a $1 \mathrm{~kg}$ mass. With an

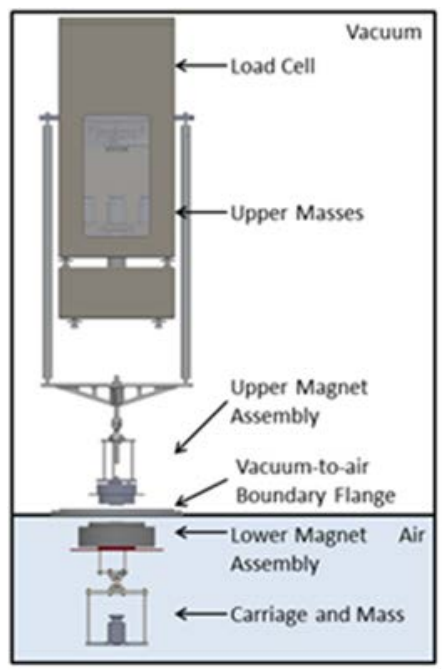

Figure 1. Schematic of the magnetic levitation principle used in the magnetic suspension mass comparator. The all-aluminium vessel has an upper chamber containing a vacuum compatible mass comparator and a lower chamber for the mass in air to be compared with the upper masses in vacuum. anticipated uncertainty of the watt balance at NIST of about 20 $\mu \mathrm{g}$, the total uncertainty would then be on the order of $30 \mu \mathrm{g}$ $(\mathrm{k}=1)$. This is acceptable for dissemination purposes and not much of a change from what NIST currently can do for customers $(\approx 20 \mu \mathrm{g}, \mathrm{k}=1)$.

In Section 2 of this paper we will discuss our method for calibrating a mass in air using a calibrated mass in vacuum with the MSMC. In Section 3 we discuss how a mass will be transported under vacuum between the watt balance and the MSMC and other facilities requiring a vacuum.

\section{NIST METHOD FOR TRANSFERRING VACUUM MASS CALIBRATIONS TO AIR}

\subsection{Vacuum chamber and mass comparator}

The apparatus is housed in two separate aluminium chambers mounted above one another as shown in Figures 1 to 3 . The upper chamber will house the mass comparator and will be kept under vacuum while the lower chamber will normally be kept at atmospheric pressure. These chambers are constructed out of non-ferromagnetic aluminium so that stray magnetic fields from the magnetic suspension system will not

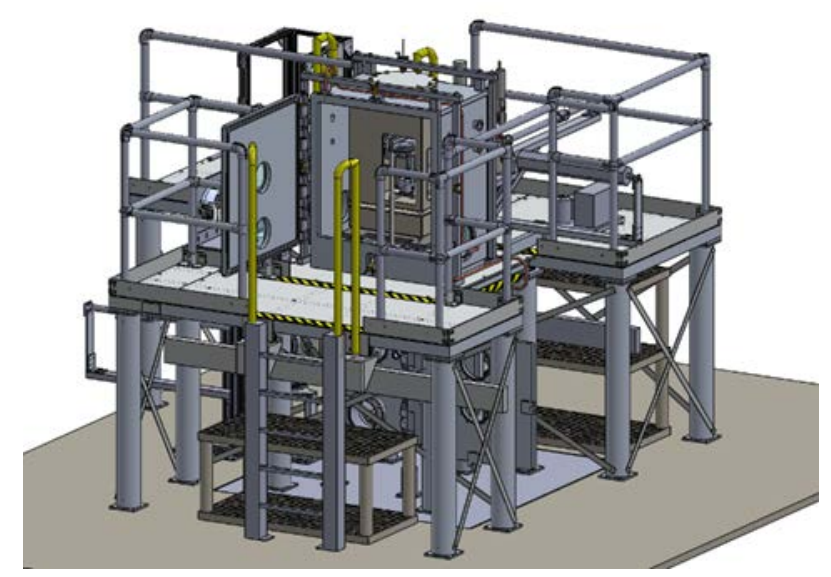

Figure 2. Illustration of magnetic suspension mass comparator facility with vacuum door opened.

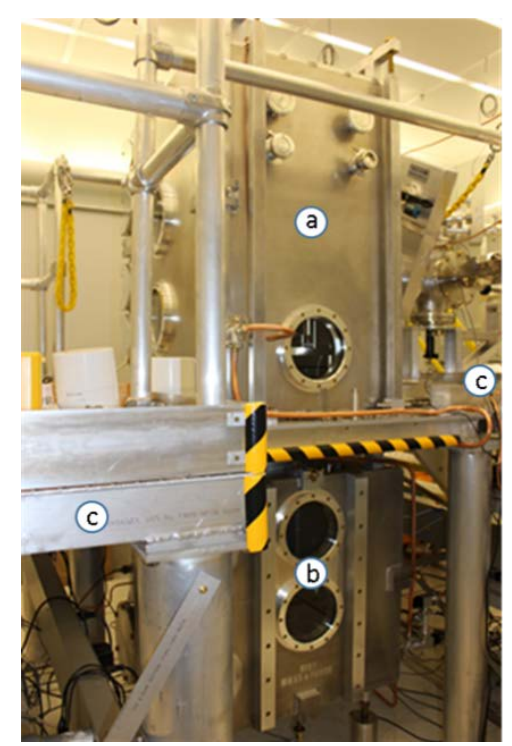

Figure 3. Magnetic Suspension Mass Comparator. a.) Upper Vacuum Chamber, b.) Lower (atmospheric) chamber, c.) raised access platforms. 
couple to the walls and interfere with the mass measurements. The chambers are rectangular in shape with the upper and lower chambers being $88 \mathrm{~cm} \times 67 \mathrm{~cm} \times 111 \mathrm{~cm}$ and $77 \mathrm{~cm} \times$ $67 \mathrm{~cm} \times 80 \mathrm{~cm}$, respectively. Large rectangular doors provide easy access to components in the vacuum chamber when necessary. Although a maximum pressure of $0.1 \mathrm{~Pa}$ would be suitable for removing buoyancy corrections, other factors such as the adsorption of water on to the masses require a lower target pressure on the order of $\left(10^{-3}\right.$ to $\left.10^{-4}\right) \mathrm{Pa}$ [10]. A magnetically levitated turbo pump produces an effective pumping rate of $134 \mathrm{l} / \mathrm{s}$ on the upper vacuum chamber. The ports and windows are sealed with fluoropolymer elastomer orings. The large door seals have a differentially-pumped dual oring design [11]. Although normally filled with air, the lower chamber can also be evacuated or filled with an inert gas.

Figure 3 shows the assembled vacuum chambers housing the MSMC. Two raised platforms on opposite sides of the apparatus provide access to the upper vacuum chamber. The chambers are mounted on a concrete pillar underneath the floor in order to vibrationally isolate the apparatus from the rest of the room, including the raised access platforms. The laboratory has a specified temperature and humidity stability to within $\pm 0.01{ }^{\circ} \mathrm{C}$ and $\pm 1 \%$ respectively.

The high precision commercial mass comparator $(10 \mathrm{~kg}$ capacity with $0.010 \mathrm{mg}$ resolution) is mounted inside the upper vacuum chamber. It includes a mass turntable which can hold up to 3 separate masses under vacuum for comparisons. In addition to the magnetically suspended weighing pan, the lower chamber also contains another mass turntable which can hold up to 4 masses in air for comparisons.

\subsection{Suspension system}

Two samarium cobalt $(\mathrm{SmCo})$ permanent disk $(3.81 \mathrm{~cm}$ diameter) magnets with a residual field of $1.16 \mathrm{~T}$ provide the necessary magnetic field to suspend both the carriage and mass in air. According to Earnshaw's theorem it is not possible to stably suspend an object with only ferrimagnets [12]. Therefore, dynamic control is required and is provided by a solenoid (75 $\Omega$ ) wound around the upper magnet [13]. A proportionalintegral-derivative (PID) control algorithm is used to stabilize the suspended mass [14]. The PID control system operates with a field programmable gate array (FPGA) that can operate independently of the computer control system. The position of the suspended mass is first determined using a Hall probe mounted on the vacuum-to-air boundary flange between the two magnets. This provides the feedback signal to the PID circuit to stabilize the suspended mass by modifying the current sent to the solenoid.

Mass comparisons with an "A-B-B-A" cycle are performed between masses in the upper vacuum chamber and the lower air chamber. During all measurements the carriage in the lower chamber is suspended, even when there is no mass on this pan. In this way, the mass of the lower weighing pan and associated suspension and support structures will cancel out of the comparison between the vacuum and air masses. In order to insure that the weighing pan is not dropped, it is not suspended when masses are transferred on/off the weighing pans.

\subsection{Gravitational field gradient}

One significant difference between the magnetic suspension measurements and a typical mass comparison measurement is that the two masses in the suspension system are being compared at different heights. The local vertical gravitational gradient is therefore used to correct the value of $g_{n}$ used in the mass comparison calculations. The gravitational gradient in the room which will house the magnetic suspension apparatus was measured with a portable commercial relative gravimeter. The gradient was measured both before and after the installation of vacuum chambers and access platforms [15]. The final results are shown in Figure 4. The masses in the MSMC will be separated vertically by $(1.082 \pm 0.001) \mathrm{m}$ and located at the corresponding points $\mathrm{A}$ and $\mathrm{C}$ in the figure. The resulting gravitational gradient is $-2.74 \times 10^{-6} \mathrm{~s}^{-2}$ and would result in a correction on the order of $300 \mu \mathrm{g}$.

\section{VACUUM MASS TRANSPORT}

\subsection{Mass transport vehicle}

NIST will realize the new kilogram with the NIST-4 watt balance [16]. The watt balance and the magnetic suspension system are located in different laboratories at NIST and it is necessary to transport masses in vacuum between the two apparatus. This will be accomplished with a mass transport vehicle (MTV) which is essentially a mobile vacuum chamber (see Figure 5). It is built mainly out of copper-gasket-sealed, stainless steel, vacuum components. It is not necessary to use non-ferromagnetic components since the MTV will not be attached to the magnetic suspension apparatus during

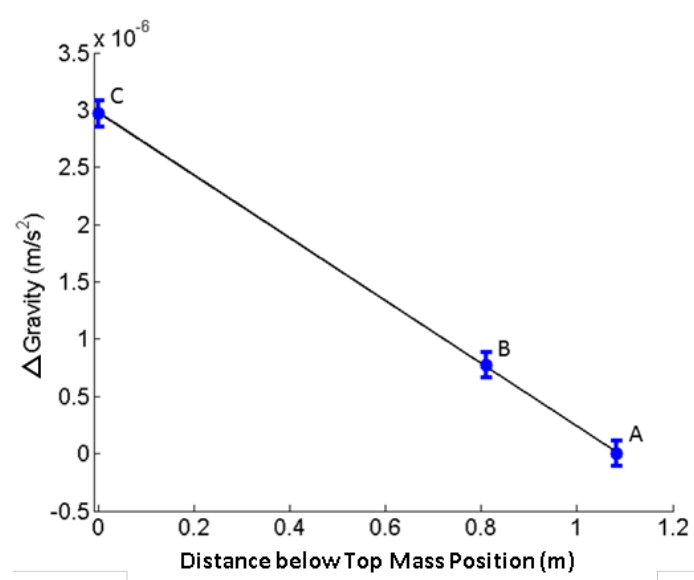

Figure 4. Variation of gravity vs. height in the magnetic suspension laboratory. the air and vacuum masses will be located at the positions corresponding to data points $A$ and $C$ respectively. Uncertainty bars represent $1 \sigma$ of the measurements.

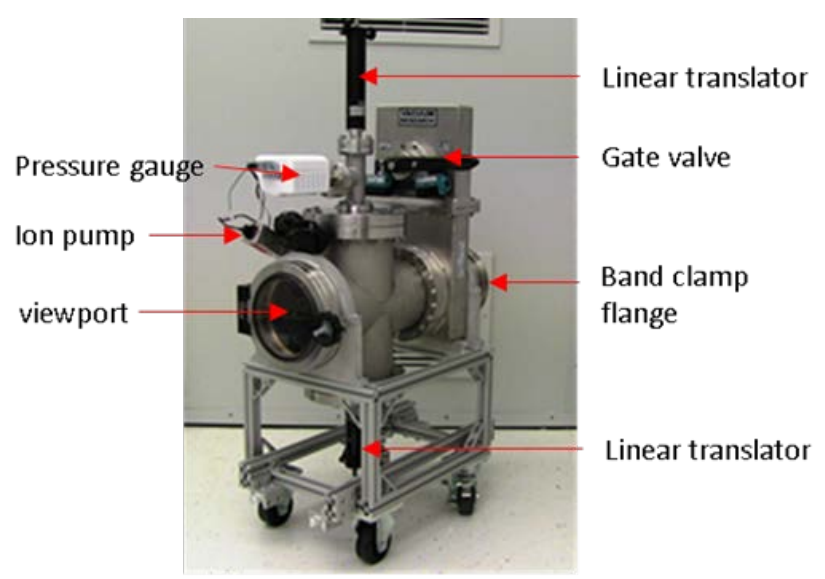

Figure 5. Mass transport vehicle. 
measurements. Only the gate valve has o-ring seals. The MTV connects to the other vacuum chambers with an ISO band clamp so that it can easily be connected and disconnected. A hot cathode ion gauge is used to monitor the vacuum and an ion pump is used to maintain the vacuum $\left(<1 \times 10^{-3} \mathrm{~Pa}\right)$ during transport. The ion pump can be battery operated to aid in keeping the pressure low during transport. Both the ion gauge and the ion pump are oriented in a way there is no direct line of sight between them and the mass to prevent possible ion sputtering onto the mass.

Figure 6 shows a cutaway view of the interior of the MTV. Masses being transported will sit on a slotted block in the center of the vehicle. Circular indents designed to match several different types of masses keep the mass from sliding sideways during transport. Three triangular wedges made from polyether ether ketone (PEEK) support the top of the mass to prevent it from tipping over. All other surfaces which could come into contact with the mass are coated with a "white bronze" tri-alloy $(\mathrm{Cu}-\mathrm{Sn}-\mathrm{Zn})$ to prevent material from being transferred to the mass. The slotted block and triangular wedges can be independently raised and lowered so that the mass can be placed on a transfer fork from the load lock which enters the MTV through the gate valve. The transfer fork when loaded with $1 \mathrm{~kg}$ and fully inserted into the MTV may sag by approximately $1 \mathrm{~cm}$. In order to vertically and horizontally align the transfer fork with the slotted block, a PEEK ramp supports the fork at the proper height.

\subsection{Load lock system}

Masses are transferred from the MTV into the magnetic suspension upper vacuum chamber through a load lock which maintains the mass under vacuum during the transfer process. The load lock is built using a 6 way cross as shown in Figure 7. The front flange of the 6 way cross is an ISO band clamp to which the MTV will be connected. Once the MTV has been attached, the load lock is evacuated with an independent turbo pump station. Once a suitable $\left(\approx 10^{-3} \mathrm{~Pa}\right)$ vacuum is obtained in

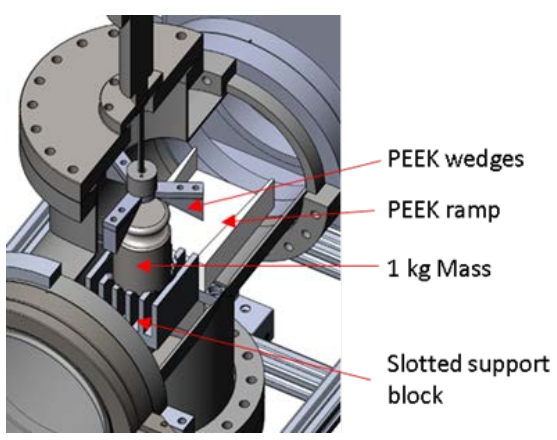

Figure 6. Illustration of the MTV interior.

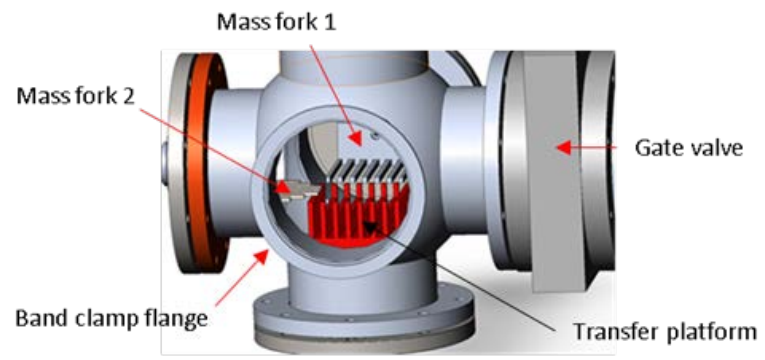

Figure 7. Illustration of a typical load lock. the load lock, the gate valves on the MTV and the load lock can be opened. Mass fork 1 in the back of the chamber, which is attached to a long linear vacuum translator, is moved into the MTV to transport the mass back to the center of the load lock. The transfer platform which is composed of an array of rectangular posts can then be moved upward to pick the mass off the mass fork. Mass fork 2, perpendicular to mass fork 1, is then moved into a position underneath the mass and between the transfer platform posts. The transfer platform can then lower the mass onto fork 2. This mass fork then transfers the mass onto the circular mass turntable of the mass comparator. The tines of mass fork 2 have different lengths so that the mating slots in the circular mass turntable for different mass positions will not intersect. Mass fork 2 is finally retracted and the gate valves closed. After venting the load lock, the MTV can be detached and removed before the mass comparison measurements are initiated. As with the MTV, all surfaces in the load lock which come into contact with the mass are coated with white bronze.

\section{CONCLUSION}

The Mass and Force Group of NIST has built a magnetic suspension system which will enable the direct comparison of a mass in air with a mass in vacuum using the same mass comparator. This is accomplished by using a magnetic suspension technique to couple a mass in the lower chamber (atmospheric pressure) to a mass comparator in the upper chamber (vacuum). The system is currently undergoing stability testing and evaluation of systematic effects. This system will become part of the NIST mise en pratique, the set of instructions for realizing and disseminating the kilogram, after redefinition. A mass transfer vehicle and load lock system will enable masses to be transported under vacuum from the NIST watt balance to the magnetic suspension apparatus and to other facilities operating under vacuum.

\section{REFERENCES}

[1] "On a new definition of the kilogram," Recommendation G 1 of the Consultative Committee for Mass and Related Quantities Submitted to the International Committee for Weights and Measures (2013), http://www.bipm.org/cc/CCM/Allowed/14/31a Recommend ation CCM G1(2013).pdf

[2] P. Richard, J. Ullrich, "Joint CCM and CCU roadmap towards the redefinition of the SI in 2018", http://www.bipm.org/utils/common/pdf/SIroadmap.pdf\#page $=3$.

[3] I. Robinson and S. Schlamminger, "The watt or Kibble balance: a technique for implementing the new SI definition of the unit of mass", Metrologia, 53 (2016) pp. A46-A74.

[4] K. Fujii, H. Bettin, P. Becker, E. Massa, O. Rienitz, A. Pramann, A. Nicolaus, N. Kuramoto, I. Busch, M. Borys, "Realization of the kilogram by the XRCD method", Metrologia, 53 (2016) pp. A19-A45.

[5] M. Stock, "Watt balance experiments for the determination of the Planck constant and the redefinition of the kilogram", Metrologia, 50 (2013) pp. R1-R16.

[6] S. Davidson, J. Berry, P. Abbott, K. Marti, R. Green, A. Malengo, L. Nielsen, "Air-vacuum transfer; establishing traceability to the new kilogram”, Metrologia, 53 (2016) pp. A95A113.

[7] "Measurements report: Air-vacuum characterization of the $1 \mathrm{~kg}$ cylindrical platinum-iridium stack C18", October 7, 2014, BIPM, Pavillon de Breteuil, F-92312 Sèvres Dedex. 
[8] K Marti, P. Fuchs, S. Russi, "Traceability of mass II: a study of procedures and materials", Metrologia, 52 (2015), pp. 89-103.

[9] Z. Jabbour, P. Abbott, E. Williams, R. Liu, V. Lee, "Linking air and vacuum mass measurements by magnetic levitation", Metrologia, 46 (2009) pp. 339-344.

[10] P. Abbott, Z. Jabour, "Vacuum technology considerations for mass metrology", J. Res. Natl. Inst. Stand. Technol., 116 (2011) pp. 689-702.

[11] A. Roth, Vacuum Technology, Elsevier Science, Amsterdam, 1990, ISBN 044488010 0, pp 416-419.

[12] S. Earnshaw, "On the nature of the molecular forces which regulate the constitution of the luminferous ether", Trans. of the Cambridge Philosophical Society, 7 (1842) pp. 97-112.
[13] G. Marsden, “Levitation!”, Nuts \& Volts, 24 (2003) pp. 58-61.

[14] C. Stambaugh, "The control system for the magnetic suspension mass comparator system for vacuum-to-air mass dissemination", ACTA IMEKO, Vol. 6, no 2, 2017.

[15] E. Mulhern, Corey Stambaugh, "Characterization of the NIST magnetic suspension mass comparator apparatus and facility", accepted for publication in NCSL International Measure, 2017.

[16] D. Haddad, F. Seifert, L.Chao, A. Cao, G. Sineriz, J. Pratt, D. Newell, S. Schlamminger, "First measurements of the flux integral with the NIST-4 watt balance", IEEE Trans. Instr. Meas., 64 (2015), pp. 1642. 